\title{
miR-362-3p targets nemo-like kinase and functions as a tumor suppressor in renal cancer cells
}

\author{
XIAOWEN ZOU ${ }^{1,2^{*}}$, JIANHUA ZHONG ${ }^{1,2^{*}}$, JIAQIANG LI ${ }^{3}$, ZHENGMING SU $^{2,4}$, YAN CHEN ${ }^{1,2}$, WANXIN DENG ${ }^{1,2}$, \\ YUCHI LI ${ }^{2,4}$, SIHENG LU ${ }^{2}$, YOUCHENG LIN ${ }^{5}$, LIYALUO ${ }^{1}$, ZESONG LI $^{1}$, ZHIMING CAI $^{1,2}$ and AIFA TANG ${ }^{1,2}$ \\ ${ }^{1}$ National-Regional Key Technology Engineering Laboratory for Clinical Application of Cancer Genomics, \\ Shenzhen Second People's Hospital, The First Affiliated Hospital of Shenzhen University, Shenzhen, Guangdong 518000; \\ ${ }^{2}$ Department of Clinical Medicine, Shantou University Medical College, Shantou, Guangdong 515063; \\ ${ }^{3}$ Department of Pediatric Urinary Surgery, Shenzhen Children's Hospital, Shenzhen, Guangdong 518000; \\ ${ }^{4}$ Guangdong and Shenzhen Key Laboratory of Male Reproductive Medicine and Genetics, \\ Peking University Shenzhen Hospital, Shenzhen, Guangdong 130012; ${ }^{5}$ Department of Urology, \\ Zhujiang Hospital of Southern Medical University, Guangzhou, Guangdong 510282, P.R. China
}

Received January 1, 2015; Accepted October 21, 2015

DOI: $10.3892 / \mathrm{mmr} .2015 .4632$

\begin{abstract}
MicroRNAs (miRNAs) have been demonstrated to exhibit abnormal expression patterns in various types of human cancer. The aim of the present study was to identify a novel tumor suppressor microRNA (miR) and investigate its physiological function and mechanism in renal cell carcinoma (RCC). The expression levels of miRNA (miR)-362-3p expres were measured in 47 pairs of RCC and adjacent normal tissue samples, using reverse transcription-quantitative polymerase chain reaction analysis. In addition, miR-362-3p was transfected into renal cancer cells to investigate its role in the regulation of cell proliferation, migration, invasion, apoptosis and cell cycle. Identification of the target gene of miR-362-3p was performed using luciferase reporter assays and western blot analyses. The results demonstrated that the expression levels of miR-362-3p were downregulated in the RCC tissue samples, compared with the adjacent normal tissue samples. The upregulation of miR-362-3p using a synthesized mimic suppressed the proliferation, migration and invasion of the renal cancer cells, and induced cell apoptosis and $\mathrm{G}_{1}$ phase arrest. Further experiments demonstrated that the overexpression of miR-362-3p resulted in decrease expression levels of
\end{abstract}

Correspondence to: Professor Aifa Tang, National-Regional Key Technology Engineering Laboratory for Clinical Application of Cancer Genomics, Shenzhen Second People's Hospital, The First Affiliated Hospital of Shenzhen University, 3002 Sungang Road, Shenzhen, Guangdong 518000, P.R. China

E-mail: tangaifa2004@163.com

${ }^{*}$ Contributed equally

Key words: microRNA-362-3p, tumor suppressor, renal cancer cells, nemo-like kinase nemo-like kinase. These results suggested that miR-362-3p functions as a tumor suppressor in RCC, and may serve as a potential molecular target in the treatment of RCC.

\section{Introduction}

Renal cell carcinoma (RCC) is the third most common type of urological cancer, and accounts for $2 \%$ of adult malignancies (1). RCC is composed of several subtypes, including clear cell, chromophobe, papillary and collecting duct carcinoma (2), categorized based on their clinical outcome and biological features. Clear cell RCC is the most common subtype, and accounts for $\sim 80 \%$ of cases of RCC (3). For all stages combined, the five-year relative survival rate is $55 \%$ (4). Despite the rapid advancement in the diagnostic and therapeutic strategies for RCC, the mortality rate has not changed significantly (5). Surgical resection remains the only definitive treatment for RCC, however, 20-40\% patients develop recurrence following curative nephrectomy (6). Therefore, it is crucial to investigate the molecular mechanism underlying the progression of RCC. MicroRNAs (miRNAs/miRs) may offer potential applications for the diagnosis, prognosis and treatment of RCC.

miRNAs are identified as an abundant class of small, non-coding RNAs, which are important in the post-transcriptional regulation of various biological processes (7-9). Currently, >1,000 miRNAs have been identified in humans. Generally, miRNAs bind to the 3' untranslated region (3'-UTR) of their target genes through imperfect complementation, and repress gene expression either by increasing mRNA degradation or by inhibiting translation (10). miRNAs suppress protein translation by binding to complementary sequences, which are predominantly located in the 3'-UTR of target messenger RNA (mRNA) (11,12). miRNAs regulate the expression levels of genes involved in several cellular processes, including cell cycle $(13,14)$, differentiation $(15,16)$ and apoptosis $(17,18)$. Furthermore, each miRNA can potentially regulate hundreds 
of mRNAs, and over one third of human genes may be miRNA targets (19-21).

There is considerable evidence indicating that miRNAs are involved in human cancer (22-24). Furthermore, it has also been suggested that deregulated miRNAs may have a tumor-suppressive or oncogenic role in different types of cancer by repressing the expression of oncogenes or tumor suppressor genes (25). Therefore, the functional identification of miRNAs has become an important area of investigation in biomedical science, however, the majority of these miRNAs have unknown functions (26). Massively parallel sequencing studies have revealed the aberrant expression of miR-362-3p in RCC $(27,28)$. Our previous study demonstrated that the expression levels of miR-362-3p are significantly downregulated in RCC (29). However, the mechanism and the association between the expression of miR-362-3p and $\mathrm{RCC}$ remain to be fully elucidated. The biological role of miR-362-3p in RCC also requires further clarification.

The aim of the present study was to analyze the expression pattern of miR-362-3p in clinical RCC tissue samples, and examine the effects of miR-362-3p on the proliferation, migration, invasion, cell cycle and apoptosis of RCC cell lines. In order to better understand the regulatory mechanism of miR-362-3p, the nemo-like kinase (NLK) target gene was validated using a luciferase reporter assay and western blot analysis.

\section{Materials and methods}

RCC clinical specimens. A total of 47 patients with RCC underwent routine surgery at the Zhujiang Hospital, Southern Medical University (Guangzhou, China) between May 2013 and April 2014. RCC tissue samples and corresponding adjacent normal renal tissue samples were collected from these 47 patients, and were immediately snap frozen in liquid nitrogen, and stored at $-80^{\circ} \mathrm{C}$ until RNA extraction. The 47 patients recruited in the present study included 34 men and 13 women, with a median age of 50 years (range, 24-84 years). None of the patients had undergone chemotherapy or radiotherapy prior to surgery. The clinical diagnosis and histology of the tumors from the patients with RCC was performed by the Cancer Center of the Southern Medical University. All tissue specimens were acquired on the basis of their availability for research objective and following a protocol approved by the ethics committee of Shenzhen Second People's Hospital (Shenzhen, China). Written informed consent was obtained from the patients prior to commencement of the present study. The clinicopathological information of the patients is shown in Table I. The disease stage of each patient was classified or reclassified according to the American Joint Committee on Cancer staging system (30).

Reverse transcription-quantitative polymerase chain reaction (RT-qPCR). To analyze the expression levels of miR-362-3p, RT-qPCR was performed using SYBR ${ }^{\circledR}$ Premix Ex Taq ${ }^{\text {TM }}$ II (Perfect Real Time; Takara Bio, Inc., Otsu, Japan) in an Mx3000P system (Agilent Technologies, Inc., Santa Clara, CA, USA). Tissue samples were homogenized and RNA was isolated using RNAios Plus total RNA extraction reagent (Takara Bio, Inc.), according to the manufacturer's protocol. Reverse transcription into cDNA was performed using a Primescript ${ }^{\mathrm{TM}}$ RT Reagent kit using gDNA Eraser (Perfect Real Time; Takara Bio, Inc.). U6 was used to normalize the data. Total RNA was reverse transcribed using the corresponding RT primer and the TaqMan MicroRNA Reverse Transcription kit (Applied Biosystems; Thermo Fisher Scientific, Inc. Waltham, MA, USA). The PCR primer for mature miR-362-3p was designed using a publicly available databases (miRBase; http://www.mirbase.org/) synthesized by Thermo Fisher Scientific, Inc. with the following sequences: miR 362-3p forward, 5'-AACACACCTATTCAA GGATTCA-3' and reverse, 5'-ACGTGACACGTTCGGAGA ATT-3'. The expression levels $\left(2^{-\Delta \Delta \mathrm{Cq}}\right)$ were normalized to those of U6 (U6 sense strand, 5'-CTCGCTTCGGCAGCACA-3', and anti-sense strand, 5'-ACGCTTCACGAATTTGCGT-3'). The $2^{-\Delta \Delta C Q}$ method was used to analyze the data, with the data presented as $\log 2$ (cancer/normal) (31).

Cell culture and miRNA transfection. ACHN and 786-O human renal carcinoma cell lines were purchased from the American Type Culture Collection (Manassas, VA, USA). Pairs of synthetic chemically modified short single or double-stranded RNA oligonucleotides, miR-362-3p mimics and an appropriate negative control (NC) were purchased from GenePharma Co. Ltd. (Shanghai, China) with the following sequences: miR 362-3p mimic strand, 5'-AACACACCTATTCAAGGA TTCA-3' and miR 362-3p negative control sense strand, 5'-TTC TCCGAACGTGTCACGTTT-3', and anti-sense strand, 5'-ACG TGACACGTTCGGAGAATT-3'. Transfection was performed using Lipofectamine ${ }^{\circledR} 2000$ reagent (Invitrogen; Thermo Fisher Scientific, Inc.), according to the manufacturer's protocol. The stable ACHN human renal cancer cell lines and 7860 cell lines were cultured in Dulbecco's modified Eagle's medium (DMEM; Gibco; Thermo Fisher Scientific, Inc.), supplemented with $10 \%$ fetal bovine serum (FBS), $50 \mu \mathrm{g} / \mathrm{ml}$ streptomycin, $50 \mathrm{U} / \mathrm{ml}$ penicillin and $2 \mathrm{mmol} / 1$ glutamine (Invitrogen). The cells were maintained in a $5 \% \mathrm{CO}_{2}$-humidified atmosphere at $37^{\circ} \mathrm{C}$. The cells $\left(\sim 8 \times 10^{3}\right.$ cells/well $)$ were seeded into a 96 -well plate $24 \mathrm{~h}$ prior to transfection, following which the ACHN and 786-O cells were transfected with the indicated quantities of the miR-362-3p mimics or miR-NC using Lipofectamine ${ }^{\circledR}$ reagent, according to the manufacturer's protocol.

Cell proliferation assay. Cell proliferation was detected using a 3-(4,5-dimethylthiazol-2-yl)-2,5-diphenyltetrazolium bromide (MTT) assay (5 mg/ml; Sigma-Aldrich, St. Louis, MO, USA). The miR-362-3p-transfected cells and miR-NC-transfected renal cancer cells were harvested and dissociated into single-cell suspensions by digestion with $0.9 \%$ trypsin for $3 \mathrm{~min}$ at $37^{\circ} \mathrm{C}$, followed by pipetting for 30 times. Cell proliferation was examined at various time points following transfection $(0,24,48$ and $72 \mathrm{~h}$ ), with $20 \mu \mathrm{l}$ MTT (5 mg/ml) added to each well, followed by incubation for $4 \mathrm{~h}$ at $37^{\circ} \mathrm{C}$. The MTT medium mixtures were subsequently discarded, following which $150 \mu \mathrm{l}$ dimethyl sulfoxide was added to each well and agitated for $15 \mathrm{~min}$ at room temperature to solubilize the crystals. The absorbance was measured at a wavelength of $490 \mathrm{~nm}$ (with $630 \mathrm{~nm}$ as the reference wavelength) using an ELISA microplate reader (HR801; Shenzhen Highcreation Technology Co., Ltd Guangdong, China). All proliferation assays were repeated as independent experiments at least three times.

Cell migration assay. Cell migration was examined using a wound-healing assay, as previously described (25). Briefly, the 
Table I. Clinicopathological characteristics of the 47 patients with renal cancer recruited in the present study.

\begin{tabular}{|c|c|}
\hline Factor & Number of cases \\
\hline \multicolumn{2}{|l|}{ Gender } \\
\hline Male & 34 \\
\hline Female & 13 \\
\hline \multicolumn{2}{|l|}{ Age } \\
\hline$\leq 50$ years & 20 \\
\hline$>50$ years & 27 \\
\hline \multicolumn{2}{|l|}{ Pathology } \\
\hline Clear cell renal cell carcinoma & 36 \\
\hline Papillary renal cell carcinoma & 5 \\
\hline Chromophobe renal cell carcinoma & 6 \\
\hline \multicolumn{2}{|l|}{ Edmondson garaging } \\
\hline $\mathrm{I}+\mathrm{II}$ & 30 \\
\hline III+IV & 17 \\
\hline \multicolumn{2}{|l|}{ Tumor size } \\
\hline$<7 \mathrm{~cm}$ & 39 \\
\hline$\geq 7 \mathrm{~cm}$ & 8 \\
\hline \multicolumn{2}{|l|}{ Lymphatic invasion } \\
\hline Positive & 0 \\
\hline Negative & 47 \\
\hline \multicolumn{2}{|l|}{ Distant metastasis } \\
\hline Positive & 0 \\
\hline Negative & 47 \\
\hline \multicolumn{2}{|l|}{ Stage } \\
\hline T1a & 17 \\
\hline T1b & 21 \\
\hline $\mathrm{T} 2$ & 6 \\
\hline $\mathrm{T} 3$ & 2 \\
\hline $\mathrm{T} 4$ & 1 \\
\hline
\end{tabular}

ACHN and 786-O stable cell lines were plated in 6-well plates $\left(5 \times 10^{5}\right.$ cells/well) and were grown to $75-90 \%$ confluence. The cell monolayers were then transfected with $50 \mathrm{~nm}$ miR-362-3p mimics. A total of $6 \mathrm{~h}$ following transfection, an artificial homogenous wound was created onto the monolayer using a sterile $200 \mu \mathrm{l}$ tip. The cells were subsequently washed with phosphate-buffered saline (PBS) and maintained in serum-free medium. Images of the cells migrating into the wound were captured at 0 and $24 \mathrm{~h}$ using a digital camera system (DMIL LED; Leica Microsystems GmbH, Wetzlar, Germany). MIAS-2000 software (Leica Microsystems GmbH) was used to determine the migration distance. The experiments were performed independently in triplicate.

Cell invasion assay. Transwell chambers $(8 \mu \mathrm{m}$ pore size; Corning Incorporated, Corning, NY, USA) were coated with Matrigel (BD Biosciences, Franklin Lakes, NJ, USA) (15 $\mu \mathrm{g} /$ filter). The cells were seeded $\left(5 \times 10^{5}\right.$ cells/well) in serum-free medium in the upper chamber (Biocoat Matrigel invasion chamber) and the lower wells were filled with $10 \%$ FBS. The cells were allowed to migrate through the Matrigel to the lower chamber for $24 \mathrm{~h}$.
Following incubation, the cells were removed from the upper surface of the filter by scraping with a cotton swab. The cells on the lower surface of the filter were permeabilized using 1\% Triton X-100 (Sigma-Aldrich), stained with crystal violet (Sigma-Aldrich) and visualized under a microscope DMI 6000B; Leica Microsystems $\mathrm{GmbH}$ ). The numbers of cells, which penetrated through the membrane were determined by counting the mean number of cells in five randomly-selected high-power fields. The experiments were repeated as independent experiments at least three times.

Cell apoptosis assay. An Annexin V-fluorescein isothiocyanate (FITC) Apoptosis Detection kit (Invitrogen) and propidium iodide (PI; Invitrogen) were used to assess the apoptotic effect of miR-362-3p. The renal cells of the different treatment groups were suspended at a concentration of $1 \times 10^{6}$ cells $/ \mathrm{ml}$. The cell suspension was transferred to a centrifuge tube, centrifuged at $7.9 \mathrm{x} g$ and washed with PBS. The cells were then resuspended in $500 \mu \mathrm{l}$ cold $1 \mathrm{X}$ binding buffer (Invitrogen) with $5 \mu \mathrm{l}$ Annexin V-FITC, and incubated for $15 \mathrm{~min}$ at room temperature in the dark. The cells were then centrifuged for $5 \mathrm{~min}$ to remove the supernatant. The cells were then resuspended in $500 \mu \mathrm{l}$ cold binding buffer with $3 \mu \mathrm{lPI}$, incubated for $15 \mathrm{~min}$ and analyzed by flow cytometry (Navi 105; Beckman Coulter, Brea, CA, USA). Experiments were performed in three independent repeats.

Cell cycle analysis. To examine the effects of the downregulation of miR-362-3p on the cell cycle, flow cytometry was performed. For cell cycle analysis, the miR-362-3p-transfected cells were harvested $48 \mathrm{~h}$ post-transfection, and were trypsinized (Invitrogen) and fixed with ice-cold 95\% ethanol (Shanghai ExCell Biology, Shanghai, China) overnight at $4^{\circ} \mathrm{C}$. The fixed cells were stained with $50 \mathrm{mg} / \mathrm{ml}$ PI, treated with $50 \mathrm{mg} / \mathrm{ml}$ RNase and then analyzed using a flow cytometer (BD Pharmingen). Each assay was independently repeated three times.

Vector construction and luciferase reporter assay. TargetScan (http://www.targetscan.org/), miRanda (http://www.microrna. org/microrna/home.do) and PicTar (http://pictar.mdc-berlin.de/) were used to computationally predict the targets of miR-362-3p. To create a luciferase reporter construct, the 3'-UTR fragment of NLK, containing putative binding sites for miR-362-3p was inserted downstream of firefly luciferase, between the XhoI-NotI restriction sites in the 3'-UTR of the hRluc gene in the psiCHECKTM-2 luciferase vector (Promega Corp., Madison, WI, USA). Mutant 3'-UTR, which carried the mutated sequence in the complementary site for miR-362-3p, was generated using the fusion PCR method, and inserted downstream of firefly luciferase between the XhoI-NotI restriction sites in the 3'-UTR of the hRluc gene in the psiCHECKTM-2 luciferase vector. The following sequences were used: NLK 3'-UTR wild-type sequence, 5'-ATATCATTCTAACGGGTGTGTT 3' and NLK 3'-UTR mutant sequence, 5'-ATATCATTCTAACGCCACACA A-3', and synthesis was performed at GenePharma Co., Ltd. Cells grown in a 48-well plate were co-transfected with miR-362-3p and the luciferase reporter comprising the wild-type or mutant 3'-UTR of the target gene. The luciferase assay was performed as described previously (32). The cells ( $5 \times 10^{5}$ per group) were co-transfected with miRNAs and 3'-UTR or a mutant 3'-UTR 
luciferase reporter, in which the potential binding sites were manually mutated by exchanging the $\mathrm{G}$ and $\mathrm{T}, \mathrm{A}$ and $\mathrm{C}$ as a control vector. At $48 \mathrm{~h}$ post-transfection, luciferase activity was measured using a Dual-Luciferase Assay kit (Promega Corp.) with a $\beta$-counter luminometer (Promega Corp.). Relative luciferase activity was calculated as the ratio of raw firefly luciferase activity and renilla luciferase activity.

$R N A$ isolation and $R T-q P C R$. Total RNA was isolated from the miR-362-3p-transfected cells using RNAiso Plus total RNA extraction reagent Takara, Bio, Inc.) according to the manufacturer's instructions. The following primers were used: NLK, sense 5'-AGCCGTCATTACAGCAAT-3' and antisense 5'-TATTCTTCGTCCTAGTAGTTCTG-3'; GAPDH, sense 5'-TGGCCTTCCGTGTTCCTAC-3' and antisense GAPDH 5'-GAGTTGCTGTTGAAGTCGCA-3'. For the detection of NLP or GAPDH mRNA, a total of $1 \mu \mathrm{g}$ mRNA was reverse-transcribed into cDNA using a One Step PrimeScript ${ }^{\mathrm{TM}}$ miRNA cDNA Synthesis kit (Takara, Bio, Inc.). qPCR was performed using SYBR ${ }^{\circledR}$ Premix Ex Taq ${ }^{\mathrm{TM}}$ II (Perfect Real Time; Takara Bio, Inc.) in a Quant Studio ${ }^{\mathrm{TM}}$ system (Agilent Technologies, Inc., Santa Clara, CA, USA), according to the manufacturer's instructions with U6 or GAPDH as an internal reference. The 20- $\mu 1$ reaction mixture contained $10 \mu \mathrm{l} 2 \mathrm{X}$ QuantiTect SYBR Green PCR Master mix, $2 \mu \mathrm{l} 10 \mathrm{X}$ miScript Universal Primer, $0.4 \mu \mathrm{l}$ specific microRNA primer, $1 \mu \mathrm{l}$ cDNA template and RNase-free water. The thermocycling conditions were as follows: $95^{\circ} \mathrm{C}$ for $15 \mathrm{~min}$, followed by 40 cycles of $94^{\circ} \mathrm{C}$ for $15 \mathrm{sec}, 55^{\circ} \mathrm{C}$ for $30 \mathrm{sec}$ and $72^{\circ} \mathrm{C}$ for $30 \mathrm{sec}$. The expression levels of miR-362-3P and NLK were quantified using the $2^{-\Delta \Delta \mathrm{Cq}}$ method. The RT-qPCR primers were synthesized by Thermo Fisher Scientific, Inc. with the sequences as follows: NLK forward, 5'-AGCCGTCATTACAGCAAT-3' and reverse, 5'-TATTCTTCGTCCTAGTAGTTCTG-3'; GAPDH forward, 5'-TGGCCTTCCGTGTTCCTAC-3' and reverse, 5'-GAGTTG CTGTTGAAGTCGCA-3'; miR 362-3p forward, 5'-AACACA CCTATTCAAGGATTCA-3' and reverse, 5'-ACGTGACAC GTTCGGAGAATT-3'; U6 forward, 5'-CTCGCTTCGGCA GCACA-3' and reverse, 5'-ACGCTTCACGAATTTGCGT-3'.

Western blot analysis. The miR-362-3p-transfected cultured cells were harvested, and total protein was extracted using radioimmunoprecipitation assay lysis buffer supplemented with proteinase/phosphatase inhibitors (Thermo Fisher Scientific, Inc.). The protein concentration was determined and equilibrated using a Pierce Bicinchoninic Acid Protein Assay kit (Thermo Fisher Scientific, Inc.). Equivalent quantities of protein $(30 \mu \mathrm{g})$ were separated by $10 \%$ SDS-PAGE (Invitrogen) and transfected onto pre-wetted polyvinylidene fluoride membranes (Millipore, Billerica, MA, USA). The membrane was blocked in $5 \%$ non-fat milk for $1 \mathrm{~h}$ and incubated overnight at $4^{\circ} \mathrm{C}$ with primary antibody. On the following day, the membranes were washed three times in Tris-buffered saline with Tween 20 (TBS-T; Beijing Solarbio Science Technology Co., Ltd., Beijing, China) and incubated with horseradish peroxidase-conjugated secondary antibody in TBS-T for $1 \mathrm{~h}$ at room temperature. An enhanced chemiluminescence kit (Millipore) was used to detect the secondary antibody. The expression levels of NLK were analyzed using mouse polyclonal anti-NLK antibody (cat. no. sc-48361; dilution 1:1,000; Santa Cruz Biotechnology,
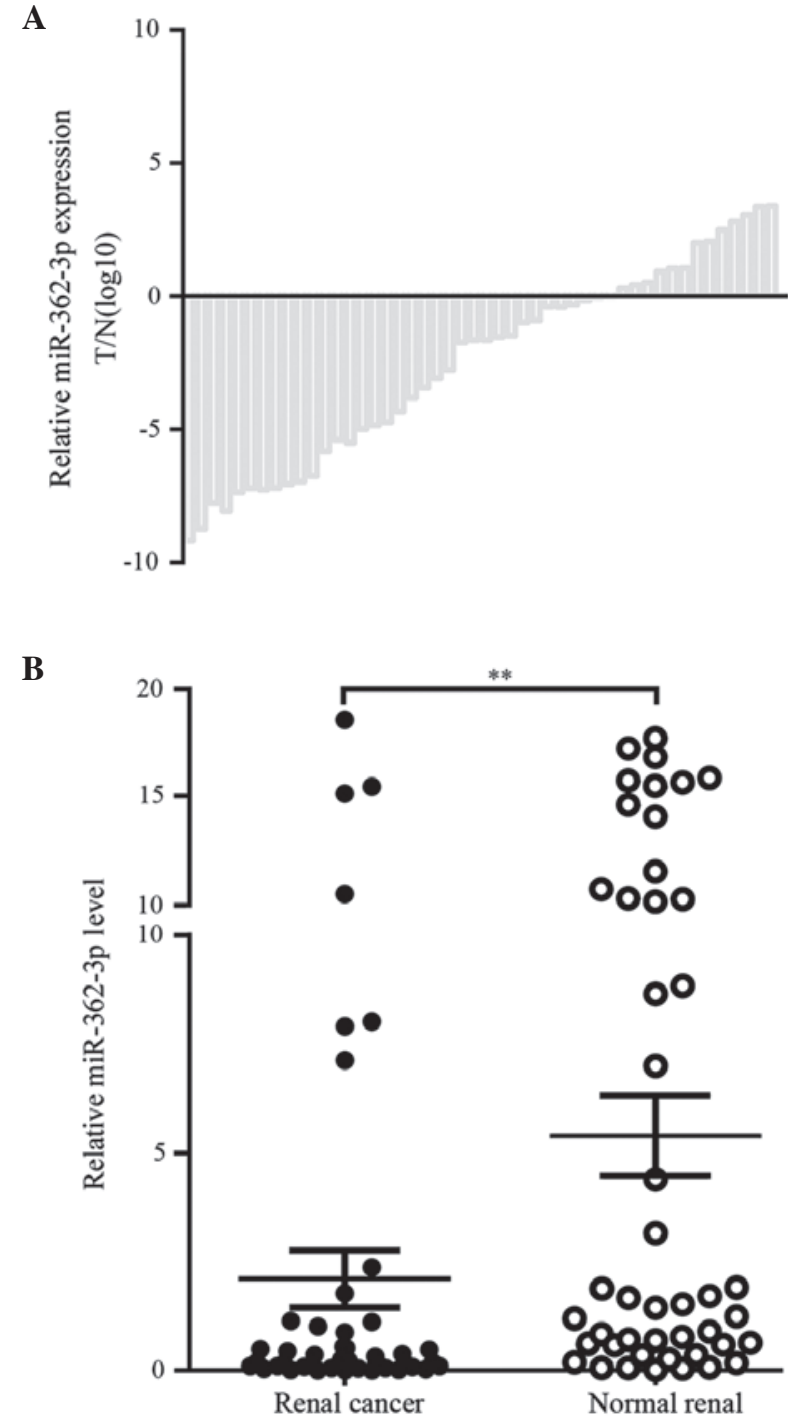

Figure 1. miR-362-3p is suppressed in renal cancer tissue samples. (A) Relative expression levels of miR-362-3p in 47 pairs of renal cancer and adjacent non-cancerous tissue samples. (B) Expression levels of miR-362-3p in renal cancer tissue samples, compared with adjacent non-cancerous tissue samples. U6 was used as an internal control. The data were analyzed using the $2^{-\Delta \Delta \mathrm{Cq}}$ method. ${ }^{* *} \mathrm{P}<0.01$. miR, microRNA; TN, Tumor/Normal.

Inc., Dallas, TX, USA), and rabbit anti-mouse polyclonal anti- $\beta$-tubulin antibody (cat. no. ab59680; dilution 1:10,000; Abcam, Cambridge, MA, USA) was used to detect the expression levels of $\beta$-tubulin, which was used as endogenous control to normalize the expression levels of NLK.

Statistical analysis. The data are presented as the mean \pm standard deviation. Data analysis was performed using Student's t-test to determine the significance between two variables. The results were analyzed using SPSS 12.0 (SPSS, Inc., Chicago, IL, USA). $\mathrm{P}<0.05$ was considered to indicate a statistically significant difference. All experiments were performed in triplicate.

\section{Results}

Expression of miR-362-3p is downregulated in $R C C$. To investigate the role of miR-362-3p in RCC development, the expression levels of miR-362-3p were examined in 47 pairs 
A
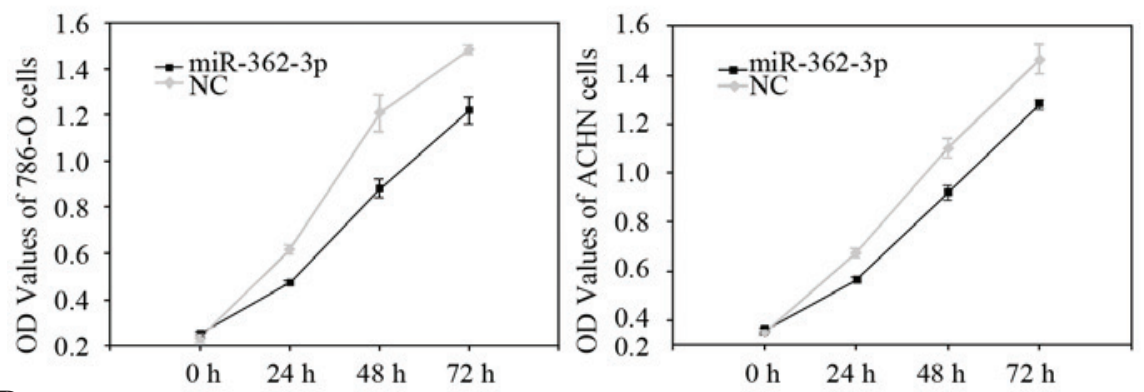

B

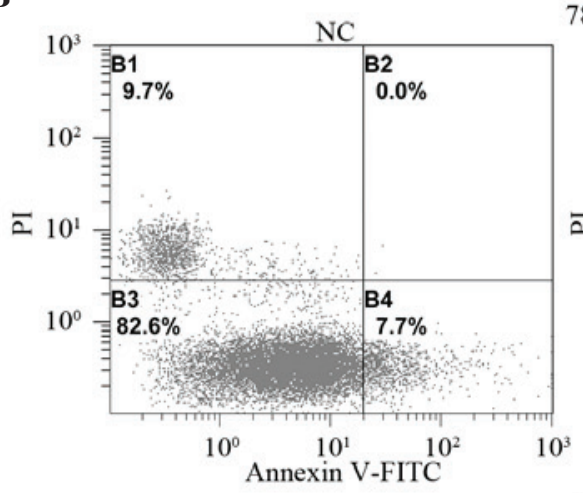

$786-\mathrm{O}$
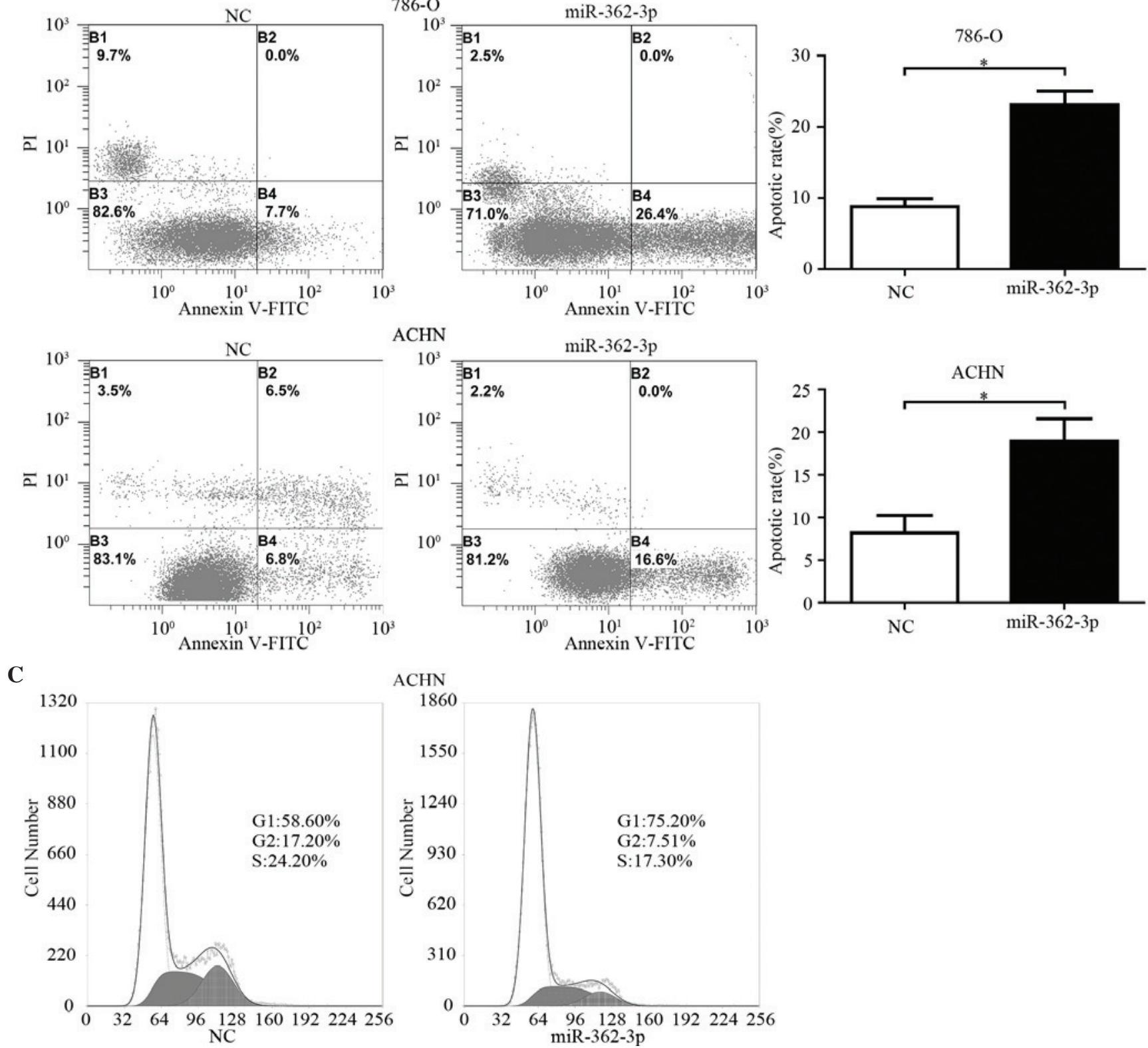

Figure 2. Effects of miR-362-3p on cell proliferation, apoptosis and cell cycle in renal cancer cells. (A) Cell proliferation was measured using a 3-(4,5-dimethylthiazol-2-yl)-2,5-diphenyltetrazolium bromide assay at for various time points. miR-362-3p inhibited 786-O and ACHN cell proliferation. (B) Cell apoptosis was analyzed using flow cytometric analysis following Annexin V-fluorescein isothiocyanate double-labeling. miR-362-3p induced apoptosis of the 786-O and ACHN cells. (C) Cell cycle distribution was determined using flow cytometry. The overexpression of miR-362-3P induced significant $\mathrm{G}_{1}$ phase arrest in the ACHN cells. All experiments were performed in triplicate, and values are expressed as the mean \pm standard deviation of three independent experiments ${ }^{*} \mathrm{P}<0.05$. miR, microRNA; PI, propidium iodide; FITC, fluorescein isothiocyanate; OD, optical density.

of RCC and adjacent normal kidney tissue samples using RT-qPCR. The decrease in the expression of miR-362-3p in the RCC tissue samples was more marked, compared with that in the adjacent normal kidney tissue samples (Fig. 1A and B). The expression levels of miR-362-3p were decreased in 35 tissue samples (74.5\%), with an overall average of $44.9 \%$ downregulation $(\mathrm{P}=0.005)$. These results suggested that miR-362-3p may act as tumor suppressor in RCC. 
A
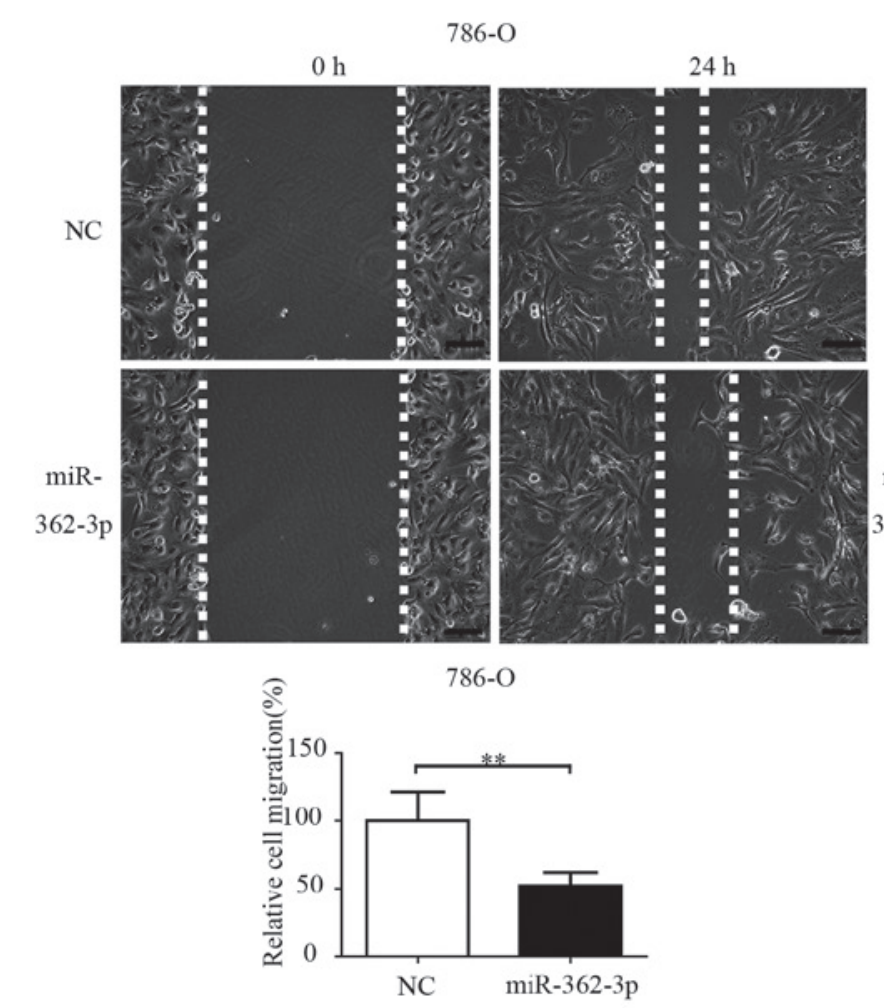

C

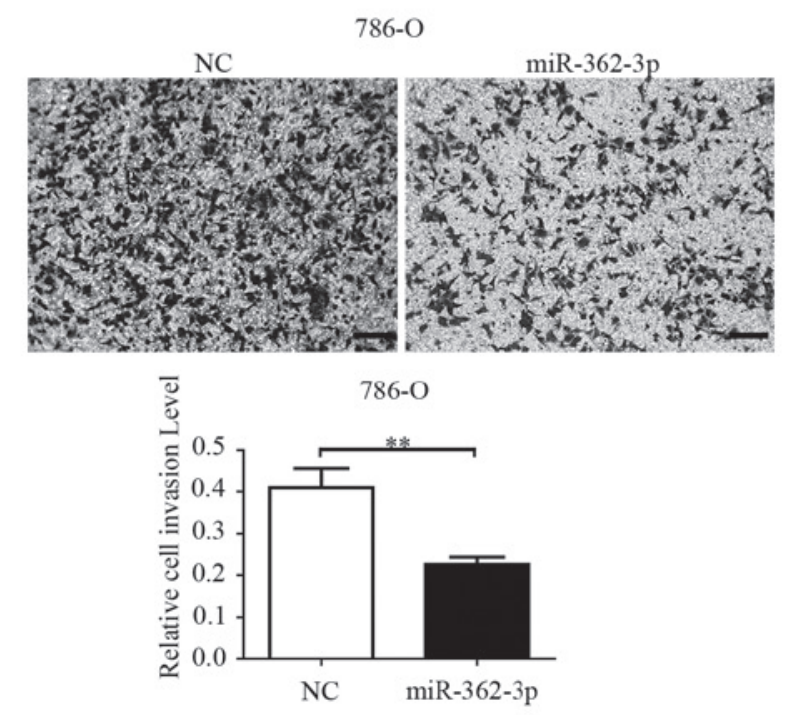

B
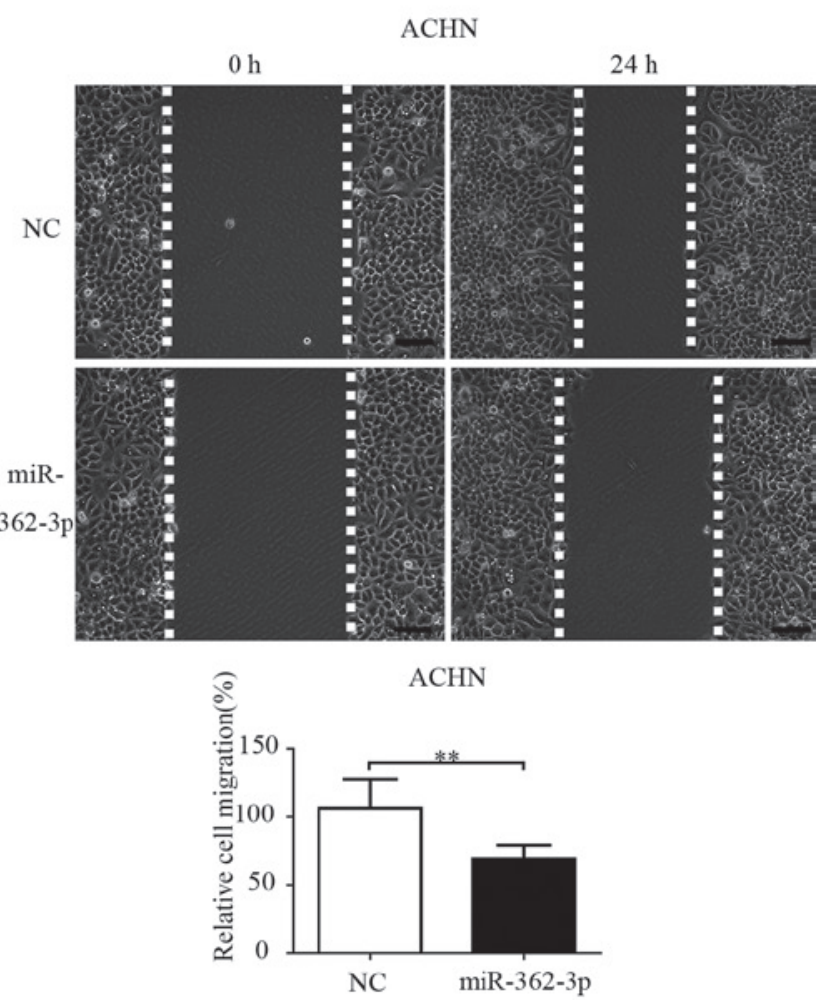

D
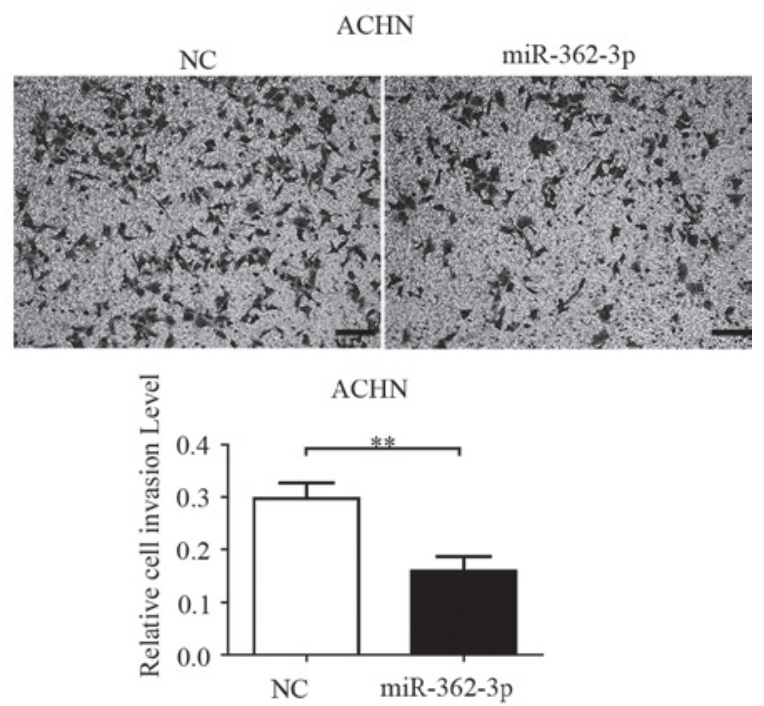

Figure 3. Effects of miR-362-3p on cell migration and invasion in renal cancer cells. (A) miR-362-3p inhibited the migration of the 786-O cells. (B) miR-362-3p inhibited the migration of the ACHN cells. (C) miR-362-3p inhibited the invasion of the 786-O cells. (D) miR-362-3p inhibited the invasion of the ACHN cells. All images are representative of three experiments (scale bar, $100 \mu \mathrm{m}$ ). Values are expressed as the mean \pm standard deviation. ${ }^{* *} \mathrm{P}<0.01$. miR, microRNA; NC, negative control.

Effects of miR-362-3p on cell proliferation, apoptosis and cell cycle in RCC cell lines. To assess the biological role of miR-362-3p in RCC, miR-362-3p mimics or NC were transfected into 786-O and ACHN cells. The MTT assay demonstrated that the relative cell proliferation in the miR-362-3p-transfected cells decreased significantly by $23.1 \%$ (24 h), 27.1\% (48 h) and $17.6 \%(72 \mathrm{~h})$ in the $786-\mathrm{O}$ cells $(\mathrm{P}<0.001)$. In the ACHN cells, the inhibition rates of cellular proliferation were $23.2 \%(24 \mathrm{~h})$, $20.2 \%$ (48 h) and 14.6\% (72 h), respectively ( $<<0.001$; Fig. 2A).

The rates of apoptosis in the 786-O and ACHN cells were analyzed using flow cytometry using miR-362-3p mimics or
NC (Fig. 2B). As shown in Fig. 2B, miR-362-3p significantly promoted apoptosis of the 786-O cells, compared with the NC (24.9, vs. $7.9 \%$, respectively; $\mathrm{P}=0.003$ ) and of the ACHN cells (18.3, vs. $7.85 \%$, respectively; $\mathrm{P}=0.021$ ).

Inhibition of cell growth in cancer cells is generally associated with cell cycle arrest. Therefore, a cell cycle assay was performed using flow cytometry with the miR-362-3p mimics or NC (Fig. 2C). A significant increase in the percentage of cells in the $\mathrm{G}_{1}$ phase to $75.2 \%$, was observed in the miR-362-3p-transfected renal cancer cells, compared with $58.6 \%$ in the miR-NC-transfected cells. These results 
A
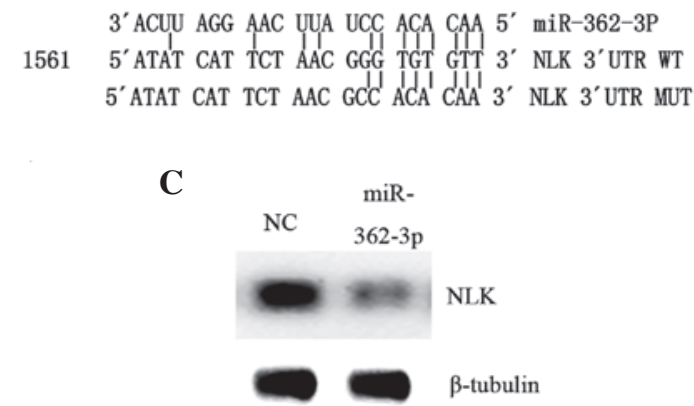

D

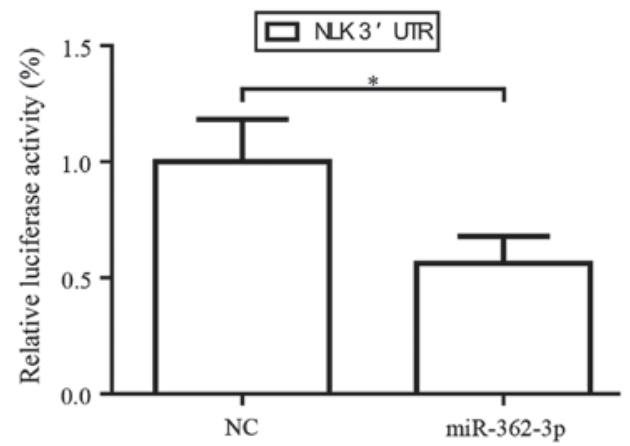

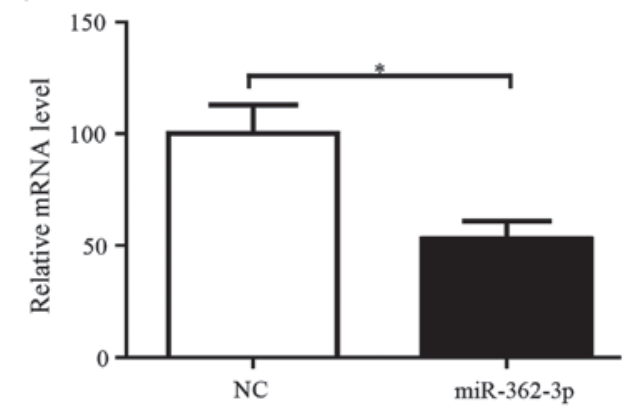

$\mathbf{E}$

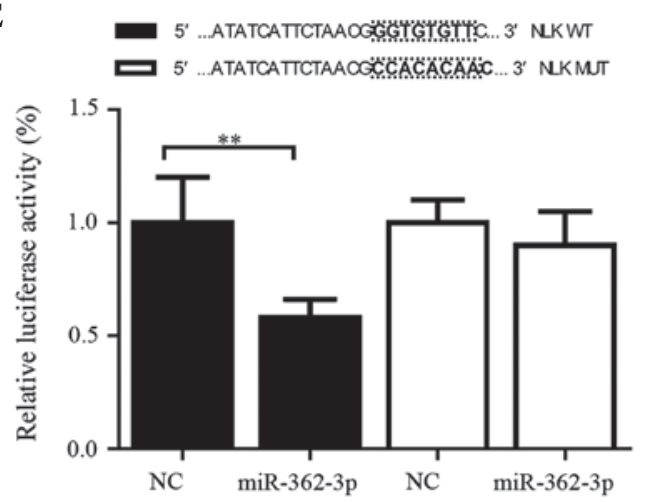

Figure 4. NLK is a target gene of miR-362-3p. (A) NLK 3'-UTR sequence and complementary miR-362-3p binding site. The mutated sequence is shown at the bottom. (B) miR-362-3p reduced the mRNA expression levels of NLK. (C) Western blot analysis was performed to detect the expression levels of NLK and $\beta$-tubulin following transfection with miR-362-3p. (D) Luciferase reporter constructs containing the 3'-UTR of NLK, together with miR-NC or miR-362-3p, were transfected into the ACHN cells. The relative levels of firefly luciferase activity were normalized with those of Renilla luciferase and measured $48 \mathrm{~h}$ following transfection. (E) Fragments of NLK 3'-UTR ( 22 nucleotides long), containing wild-type potential binding sites were synthesized. Mutated-type fragments were generated by exchanging the T and $\mathrm{A}, \mathrm{G}$ and $\mathrm{C}$ on the putative binding sites. The sequences of these two fragments are shown in the upper panels. The experiments were repeated as independent experiments at least three times. Data are presented as the mean \pm standard deviation. ${ }^{*} \mathrm{P}<0.05$ and ${ }^{* *} \mathrm{P}<0.01$. miR, microRNA; NC, negative control; UTR, untranslated region; WT, wild-type; Mut, mutant.

suggested that miR-362-3p may lead to $\mathrm{G}_{1}$ cell cycle arrest in RCC cells.

Effects of miR-362-3p on cell migration and invasion in $R C C$ cell lines. To investigate whether miR-362-3p had an effect on facilitating renal cancer cell migration, migration was assessed using a wound healing assay in the 786-O and ACHN cells. As shown in Fig. 3A and B, cell migration was significantly reduced in the cells transfected with miR-362-3p, compared with those in the miR-NC group (40 and 50\%, respectively; $\mathrm{P}<0.05$; Fig. $3 \mathrm{~A}$ and $\mathrm{B}$ ). These results suggested that $\mathrm{miR}-362-3 \mathrm{p}$ has a negative effect on cellular migration.

As invasion is an important characteristic of malignant tumors, the present study investigated the effects of miR-362-3p on tumor invasion in the 786-O and ACHN cell lines. The invasion assay demonstrated that cell invasion was significantly inhibited in the miR-362-3p-transfected cells, compared with the NC-transfected cells. The number of invading cells decreased by $49.8 \%(\mathrm{P}<0.001)$ in the $786-\mathrm{O}$ cells and by $55.3 \%(\mathrm{P}<0.001)$ in the ACHN cells, suggesting that miR-362-3p inhibited the invasive potential of the renal cancer cells (Fig. 3C and D).

$N L K$ is a target gene of miR-362-3p. TargetScan (http://www.targetscan.org/), miRanda (http://www.microrna. org/microrna/home.do) and PicTar (http://pictar.mdc-berlin. $\mathrm{de} /$ ) were used to computationally predict the targets of miR-362-3p. The cancer-promoting gene, NLK, was predicted to be one potential target (Fig. 4A). The potential binding site of miR-362-3p (position 854-860) was predicted to be the NLK 3'-UTR. The mRNA and protein expression levels of NLK were quantified in the renal cancer cells following transfection with $\mathrm{miR}-362-3 \mathrm{p}$ mimics. The mRNA and protein expression levels of NLK were decreased following transfection (Fig. 4B and C). As shown in Fig. 4D and E, transfection of the renal cancer cells with the miR-362-3p mimics in the wild-type 3'-UTR (pLuc-NLK 3'-UTR-wild) vector significantly reduced luciferase activity, compared with the NC $(\mathrm{P}<0.05)$. However, transfection of the renal cancer cells with the miR-362-3p mimics in the mutant 3'-UTR (pLuc-NLK 3'-UTR-mut) vector had no effect on luciferase activity, compared with the control inhibitor $(\mathrm{P}>0.05)$. These results indicated that miR-362-3p downregulated the luciferase activity of the reporter. The data also identified NLK as a target gene of miR-362-3p, and identified the sites of interaction in the 3'-UTR of NLK.

\section{Discussion}

Despite significant improvements in cancer therapy, RCC is relatively resistant to traditional cancer treatments, including 
radiotherapy, immunotherapy and chemotherapy (33). Therefore, the identification of novel, more accurate and prophetic prognostic markers, which are not currently included in conventional staging systems, aim to improve the prognosis of patients with RCC. miRNAs are aberrantly expressed in several types of cancer and appear to have diagnostic and prognostic significance. Dysregulation of miRNAs is associated with various human diseases, particularly cancer (21). It has been established that miRNAs regulate various important cellular processes, including proliferation, cell cycle, differentiation and tumor formation (34).

Previous studies have demonstrated the presence of aberrant miRNA expression in human cancer (25), including RCC (35), a number of which function as tumor suppressor genes or oncogenes (36). Furthermore, next-generation sequencing techniques have provided further insights into the genetic basis of RCC (37). Consequently, the identification of novel biomarkers, particularly for patients with RCC is urgently required. In our previous study, massively parallel sequencing revealed the downregulation of miR-362-3p in ccRCC (29). The downregulation of miR-362-3p and its functional analysis in colon cancer cell lines has also suggested a tumor-suppressing role for miR-362-3p (38). However, the association between the expression of miR-362-3p and RCC remains to be elucidated and requires further clarification. The present study investigated the downregulation of miR-362-3p in RCC tissues, compared with normal tissues. Based on the results, it was hypothesized that miR-362-3p may have an important role as a tumor suppressor in kidney cancer. The results demonstrated that the ectopic expression of miR-362-3p caused inhibition of proliferation, invasiveness and migration, and increased cell apoptosis in the RCC cells. These results confirmed the anticancer effect of miR-362-3p, and provide sufficient evidence to support the tumor suppressor role of miR- 362-3p in RCC (39).

Although the results of the present study demonstrated the importance of miR-362-3p as a tumor suppressor in RCC, the precise molecular mechanisms underlying its function remain to be elucidated. Mature miRNAs regulate the expression of target genes at the post-transcriptional level via the degradation of transcripts and inhibition of translation though binding to the 3'-UTR of target mRNA. To acquire a better understanding of the tumor suppressive effects of miR-362-3p in renal tumor formation, the analysis of miR-362-3p-predicted targets was performed using the following algorithms: MiRanda (http://www.microrna.org/microrna/home. do), PicTar (http://pictar.mdc-berlin.de/) and TargetScan (http://targetscan.org/). The binding sites of miR-362-3p were situated on the NLK 3'-UTR, determined using a luciferase reporter assay. Furthermore, the results of the present study demonstrated that overexpression of miR-362-3p downregulated the mRNA and protein expression levels of NLK.

NLK is a promutogenic nemo-like kinase, and is a classic mediator of the Wnt/ $\beta$-catenin signaling pathway (40). NLK belongs to the threonine protein kinase super family and is important in the apoptosis of cancer cells. Previous studies have also shown that NLK is critical in tumor occurrence and development, and is therefore becoming the subject of current studies. For example, studies have revealed that the expression levels of NLK in cancer tissue samples are significantly higher, compared with corresponding normal tissue samples in hepatocellular carcinoma and ovarian cancer $(41,42)$. In 2003, Yasuda et al demonstrated that overexpression of NLK may have targets other than TCF in the induction of apoptosis in human colon carcinoma cells (43). In 2009, Emami et al (44) revealed that overexpression of NLK results in more pronounced induction of apoptosis in androgen receptor (AR)-expressing LNCaP cells, compared with AR-negative PC-3 cells. In 2010, Jung et al (45) suggested that the expression level of NLK in cancer tissue samples are significantly higher, compared with those in corresponding normal liver tissue samples in hepatocellular carcinoma, and also demonstrated that NLK may be involved in promoting the growth of hepatoma cells via promoting cell cycle progression. In 2011, Cui et al (46) reported that NLK induces apoptosis in glioma cells via the activation of caspases, therefore, NLK may serve as a useful independent prognostic indicator for glioma. In 2012, Stevens et al (42) demonstrated that the expression levels of NLK are higher in normal ovarian tissue samples, compared with ovarian cancer tissue samples. In the present study, the data showed that miR-362-3p regulated the expression of NLK by directly binding to its 3'-UTR, suggesting that miR-362-3p may have tumor suppressive functions by regulating oncogenic genes in RCC. The overexpression of miR-362-3p in RCC may lead to the downregulation of NLK, which subsequently provides a growth and expansion advantage during renal carcinogenesis.

In conclusion, the present study demonstrated that miR-362-3p was frequently reduced in clinical tissue specimens of RCC. miR-362-3p exerted suppressive effects on tumor proliferation, migration and invasion by directly or indirectly regulating targeted genes. To the best of our knowledge, the present study provided the first evidence that miR-362-3P may act as a tumor suppressor in RCC. These findings may be pivotal in RCC oncogenesis, and provide evidence supporting the use of miRNAs as a novel approach for the detection, prevention and treatment of RCC.

\section{Acknowledgements}

The present study was supported by the National Natural Science Foundation of China (grant nos. 81170613 and 81270740) and the Shenzhen Basic Research knowledge Innovation Program (grant no. JCYJ20140416180 323426).

\section{References}

1. Grange C, Collino F, Tapparo M and Camussi G: Oncogenic micro-RNAs and renal cell carcinoma. Front Oncol 4: 49, 2014.

2. Lopez-Beltran A, Carrasco JC, Cheng L, Scarpelli M, Kirkali Z and Montironi R: 2009 update on the classification of renal epithelial tumors in adults. Int J Urol 16: 432-443, 2009.

3. Rini BI, Campbell SC and Escudier B: Renal cell carcinoma. Lancet 373: 1119-1132, 2009.

4. van Spronsen DJ, de Weijer KJ, Mulders PF and De Mulder PH: Novel treatment strategies in clear-cell metastatic renal cell carcinoma. Anticancer Drugs 16: 709-717, 2005.

5. Reeves DJ and Liu CY: Treatment of metastatic renal cell carcinoma. Cancer Chemother Pharmacol 64: 11-25, 2009.

6. Janzen NK, Kim HL, Figlin RA and Belldegrun AS: Surveillance after radical or partial nephrectomy for localized renal cell carcinoma and management of recurrent disease. Urol Clin North Am 30: 843-852, 2003. 
7. Shao Y,Zhang SQ, Quan F, Zhang PF and Wu SL: MicroRNA-145 inhibits the proliferation, migration and invasion of the human TCA8113 oral cancer line. Oncol Lett 6: 1636-1640, 2013.

8. Yang L, Wang YL, Liu S, Zhang PP, Chen Z, Liu M and Tang H: miR-181b promotes cell proliferation and reduces apoptosis by repressing the expression of adenylyl cyclase 9 (AC9) in cervical cancer cells. FEBS Lett 588: 124-130, 2014.

9. Billeter AT, Barnett RE, Druen D, Polk HC Jr and van Berkel VH MicroRNA as a new factor in lung and esophageal cancer. Semin Thorac Cardiovasc Surg 24: 155-165, 2012.

10. Eulalio A, Huntzinger E and Izaurralde E: Getting to the root of miRNA-mediated gene silencing. Cell 132: 9-14, 2008.

11. Lytle JR, Yario TA and Steitz JA: Target mRNAs are repressed as efficiently by microRNA-binding sites in the 5'UTR as in the 3'UTR. Proc Natl Acad Sci USA 104: 9667-9672, 2007.

12. Wightman B, Bürglin TR, Gatto J, Arasu P and Ruvkun G: Negative regulatory sequences in the lin-14 3'-untranslated region are necessary to generate a temporal switch during Caenorhabditis elegans development. Genes Dev 5: 1813-1824, 1991.

13. Lal A, Navarro F, Maher CA, Maliszewski LE, Yan N, O'Day E, Chowdhury D, Dyk xhoorn DM, Tsai P, Hofmann O, et al: miR-24 Inhibits cell proliferation by targeting E2F2, MYC and other cell-cycle genes via binding to 'seedless' 3'UTR microRNA recognition elements. Mol Cell 35: 610-625, 2009.

14. Lerner M, Lundgren J, Akhoondi S, Jahn A, Ng HF, Akbari Moqadam F, Oude Vrielink JA, Agami R, Den Boer ML, Grandér D and Sangfelt O: MiRNA-27a controls FBW7/hCDC4-dependent cyclin E degradation and cell cycle progression. Cell Cycle 10: 2172-2183, 2011.

15. Ebert PJ, Jiang S, Xie J, Li QJ and Davis MM: An endogenous positively selecting peptide enhances mature $\mathrm{T}$ cell responses and becomes an autoantigen in the absence of microRNA miR-181a. Nat Immunol 10: 1162-1169, 2009.

16. Ventura A, Young AG, Winslow MM, Lintault L, Meissner A, Erkeland SJ, Newman J, Bronson RT, Crowley D, Stone JR, et al: Targeted deletion reveals essential and overlapping functions of the miR-17 through 92 family of miRNA clusters. Cell 132 875-886, 2008.

17. Cimmino A, Calin GA, Fabbri M, Iorio MV, Ferracin M, Shimizu M, Wojcik SE, Aqeilan RI, Zupo S, Dono M, et al: miR-15 and miR-16 induce apoptosis by targeting BCL2. Proc Natl Acad Sci USA 102: 13944-13949, 2005.

18. Curtale G, Citarella F, Carissimi C, Goldoni M, Carucci N, Fulci V, Franceschini D, Meloni F, Barnaba V and Macino G: An emerging player in the adaptive immune response: MicroRNA-146a is a modulator of IL-2 expression and activation-induced cell death in T lyzmphocytes. Blood 115 . 265-273, 2010

19. Yu Z, Ni L, Chen D, et al: Identification of miR-7 as an oncogene in renal cell carcinoma. J Mol Histol 44: 669-677, 2013.

20. Peng H, Luo J, Hao H, Hu J, Xie SK, Ren D and Rao B MicroRNA-100 regulates SW620 colorectal cancer cell proliferation and invasion by targeting RAP1B. Oncol Rep 31: 2055-2062, 2014.

21. Xie J, Chen M, Zhou J, Mo MS, Zhu LH, Liu YP, Gui QJ, Zhang L and Li GQ: miR-7 inhibits the invasion and metastasis of gastric cancer cells by suppressing epidermal growth factor receptor expression. Oncol Rep 31: 1715-1722, 2014.

22. Lewis BP, Burge CB and Bartel DP: Conserved seed pairing, often flanked by adenosines, indicates that thousands of human genes are microRNA targets. Cell 120: 15-20, 2005.

23. Babashah S and Soleimani M: The oncogenic and tumour suppressive roles of microRNAs in cancer and apoptosis. Eur J Cancer 47: 1127-1137, 2011

24. Seca H, Almeida GM, Guimarães JE and Vasconcelos MH: miR signatures and the role of miRs in acute myeloid leukaemia. Eur J Cancer 46: 1520-1527, 2010.

25. Schickel R, Boyerinas B, Park SM and Peter ME: MicroRNAs: Key players in the immune system, differentiation, tumorigenesis and cell death. Oncogene 27: 5959-5974, 2008

26. Ambros V: The functions of animal microRNAs. Nature 431: $350-355,2004$
27. Müller S and Nowak K: Exploring the miRNA-mRNA regulatory network in clear cell renal cell carcinomas by next-generation sequencing expression profiles. Biomed Res Int 2014: 948408 , 2014.

28. Osanto S, Qin Y, Buermans HP, Berkers J, Lerut E, Goeman JJ and van Poppel H: Genome-wide microRNA expression analysis of clear cell renal cell carcinoma by next generation deep sequencing. PLoS One 7: e38298, 2012.

29. Zhou L, Chen J, Li Z, Li X, Hu X, Huang Y, Zhao X, Liang C, Wang Y, Sun L, et al: Integrated profiling of microRNAs and mRNAs: MicroRNAs located on Xq27.3 associate with clear cell renal cell carcinoma. PLoS One 5: e15224, 2010.

30. Edge SB and Compton CC: The American Joint Committee on Cancer: The 7th edition of the AJCC cancer staging manual and the future of TNM. Ann Surg Oncol 17: 1471-1474, 2010.

31. Livak KJ and Schmittgen TD: Analysis of relative gene expression data using real-time quantitative PCR and the 2(-Delta Delta C(T)) Method. Methods 25: 402-408, 2001.

32. Su Z, Chen D, Zhang E, et al: MicroRNA-509-3p inhibits cancer cell proliferation and migration by targeting the mitogen-activated protein kinase kinase kinase 8 oncogene in renal cell carcinoma. Mol Med Rep 12: 1535-1543, 2015.

33. Patel C, Ahmed A and Ellsworth P: Renal cell carcinoma: A reappraisal. Urol Nurs 32: 182-190, 2012.

34. Yang SF, Hsu HL, Chao TK, Hsiao CJ, Lin YF and Cheng CW: Annexin A2 in renal cell carcinoma: Expression, function and prognostic significance. Urol Oncol 33: e11-e21, 2015.

35. Lang Y, Xu S, Ma J, Wu J, Jin S, Cao S and Yu Y: MicroRNA-429 induces tumorigenesis of human non-small cell lung cancer cells and targets multiple tumor suppressor genes. Biochem Biophys Res Commun 450: 154-159, 2014.

36. Huang Y, Dai Y, Yang J, Chen T, Yin Y, Tang M, Hu C and Zhang L: Microarray analysis of microRNA expression in renal clear cell carcinoma. Eur J Surg Oncol 35: 1119-1123, 2009.

37. Luthra R, Chen H, Roy-Chowdhuri S and Singh RR Next-generation sequencing in clinical molecular diagnostics of cancer: Advantages and challenges. Cancers (Basel) 7: 2023-2036, 2015.

38. Christensen LL, Tobiasen H, Holm A, Schepeler T, Ostenfeld MS, Thorsen K, Rasmussen MH, Birkenkamp-Demtroeder K, Sieber OM, Gibbs P, et al: MiRNA-362-3p induces cell cycle arrest through targeting of E2F1, USF2 and PTPN1 and is associated with recurrence of colorectal cancer. Int J Cancer 133 67-78, 2013

39. Van Wynsberghe PM, Chan SP, Slack FJ and Pasquinelli AE: Analysis of microRNA expression and function. Methods Cell Biol 106: 219-252, 2011.

40. Ishitani T and Ishitani S: Nemo-like kinase, a multifaceted cell signaling regulator. Cell Signal 25: 190-197, 2013.

41. Yuzugullu H, Benhaj K, Ozturk N, Senturk S, Celik E, Toylu A, Tasdemir N, Yilmaz M, Erdal E, Akcali KC, et al: Canonical wnt signaling is antagonized by noncanonical wnt5a in hepatocellular carcinoma cells. Mol Cancer 8: 90, 2009.

42. Stevens KN, Kelemen LE, Wang X, Fridley BL, Vierkant RA, Fredericksen Z, Armasu SM, Tsai YY, Berchuck A, Narod SA, et al: Common variation in nemo-like kinase is associated with risk of ovarian cancer. Cancer Epidemiol Biomarkers Prev 21: 523-528, 2012.

43. Yasuda J, Tsuchiya A, Yamada T, Sakamoto M, Sekiya T and Hirohashi S: Nemo-like kinase induces apoptosis in DLD-1 human colon cancer cells. Biochem Biophys Res Commun 308: 227-233, 2003.

44. Emami KH, Brown LG, Pitts TE, Sun X, Vessella RL and Corey E: Nemo-like kinase induces apoptosis and inhibits androgen receptor signaling in prostate cancer cells. Prostate 69: 1481-1492, 2009

45. Jung KH, Kim JK, Noh JH, Eun JW, Bae HJ, Xie HJ, Ahn YM, Park WS, Lee JY and Nam SW: Targeted disruption of Nemo-like kinase inhibits tumor cell growth by simultaneous suppression of cyclin D1 and CDK2 in human hepatocellular carcinoma. J Cell Biochem 110: 687-696, 2010.

46. Cui G, Li Z, Shao B, Zhao L, Zhou Y, Lu T, Wang J, Shi X, Wang J, Zuo G, et al: Clinical and biological significance of nemo-like kinase expression in glioma. J Clin Neurosci 18: 271-275, 2011. 